\title{
Numerical Exponential Decay to Dissipative Bresse System
}

\author{
M. L. Santos and Dilberto da S. Almeida Júnior \\ Department of Mathematics, Federal University of Pará, Augusto Corrêa Street no. 01, Belém, \\ CEP 66075-110, Pará, Brazil
}

Correspondence should be addressed to M. L. Santos, ls@ufpa.br

Received 18 February 2010; Revised 25 May 2010; Accepted 24 June 2010

Academic Editor: Wan Tong Li

Copyright (C) 2010 M. L. Santos and D. d. S. A. Júnior. This is an open access article distributed under the Creative Commons Attribution License, which permits unrestricted use, distribution, and reproduction in any medium, provided the original work is properly cited.

We consider the Bresse system with frictional dissipative terms acting in all the equations. We show the exponential decay of the solution by using a method developed by Z. Liu and S. Zheng and their collaborators in past years. The numerical computations were made by using the finite difference method to prove the theoretical results. In particular, the finite difference method in our case is locking free.

\section{Introduction and Main Results}

The circular arch problem is also known as the Bresse system (see [1] for details). Elastic structures of the arches type are objects of study widely explored in engineering, architecture, marine engineering, aeronautics and others. In particular, the free vibrations about elastic structures is a function of their natural properties and are an important subject of investigation in engineering and also in mathematics. In the field of mathematical analysis is interesting to know properties which relate the behavior of the energy associated with the respective dynamic model. For feedback laws, for example, we can ask what conditions about dynamic model must be ensured to obtain the decay of the energy of solutions in the time $t$. In this sense, the property of stabilization has been studied for dynamic problems in elastic structures translated in terms of partial differential equations. A interesting property determines that the exponential decay with few feedback laws occurs only in an particular situation (see $[2,3])$.

Regarding the numerical aspects related to elastic structures, special attention was dedicated for a numerical pathology know as locking phenomenon about transverse shear force or simply shear locking (see [4-7]). The locking phenomenon is a numerical anomaly 
that affect some numerical methods when applied in elastic structures, such as finite element methods for linear shape functions. Basically, this obstacle numerical consists in an overestimation about of the coefficient of rigidity and that obviously does not correspond to the real case. Therefore, the locking phenomenon is a numerical inconsistent. In this sense, any numerical method applied to elastic structures must be to avoid this numerical anomaly.

Following the aim idea about deformation in elastic structures, we consider the Bresse system given by equations of motion

$$
\begin{gathered}
\rho_{1} \varphi_{t t}=Q_{x}+l N \\
\rho_{2} \psi_{t t}=M_{x}-Q, \\
\rho_{1} w_{t t}=N_{x}-l Q
\end{gathered}
$$

where we use $N, Q$ and $M$ to denote the axial force, the shear force and the bending moment, respectively. These forces are stress-strain relations for elastic behavior and given by

$$
\begin{gathered}
N=\kappa_{0}\left(w_{x}-l \varphi\right), \\
Q=\kappa\left(\varphi_{x}+l w+\psi\right), \\
M=b \psi_{x} .
\end{gathered}
$$

Here $\rho_{1}=\rho A, \rho_{2}=\rho I, \kappa=k^{\prime} G A, \kappa_{0}=E A, b=E I, l=R^{-1}$ where $\rho$ is the density of material, $E$ is the modulus of elasticity, $G$ is the shear modulus, $k^{\prime}$ is the shear factor, $A$ is the cross-sectional area, $I$ is the second moment of area of the cross-section and $R$ is the radius of curvature. The functions $w, \varphi$, and $\psi$ are the longitudinal, vertical and shear angle displacements, respectively (see Figure 1).

From coupled equations (1.1) and (1.2) we obtain

$$
\begin{aligned}
\rho_{1} \varphi_{t t}-\kappa\left(\varphi_{x}+\psi+l w\right)_{x}-\kappa_{0} l\left(w_{x}-l \varphi\right)=0, & \text { in }(0, L) \times(0, T) \\
\rho_{2} \psi_{t t}-b \psi_{x x}+\kappa\left(\varphi_{x}+\psi+l w\right)=0, & \text { in }(0, L) \times(0, T) \\
\rho_{1} w_{t t}-\kappa_{0}\left(w_{x}-l \varphi\right)_{x}+\kappa l\left(\varphi_{x}+\psi+l w\right)=0, & \text { in }(0, L) \times(0, T) .
\end{aligned}
$$
see [1].

In the literature (1.3) are the equations for the theory of circular arch. For more details

In this paper we will examine the issues concerning the asymptotic stabilization of Bresse system with frictional dissipative terms given by

$$
\begin{gathered}
\rho_{1} \varphi_{t t}-\kappa\left(\varphi_{x}+\psi+l w\right)_{x}-\kappa_{0} l\left(w_{x}-l \varphi\right)+\gamma_{1} \varphi_{t}=0, \quad \text { in }(0, L) \times(0, T), \\
\rho_{2} \psi_{t t}-b \psi_{x x}+\kappa\left(\varphi_{x}+\psi+l w\right)+\gamma_{2} \psi_{t}=0, \quad \text { in }(0, L) \times(0, T), \\
\rho_{1} w_{t t}-\kappa_{0}\left(w_{x}-l \varphi\right)_{x}+\kappa l\left(\varphi_{x}+\psi+l w\right)+\gamma_{3} w_{t}=0, \quad \text { in }(0, L) \times(0, T),
\end{gathered}
$$




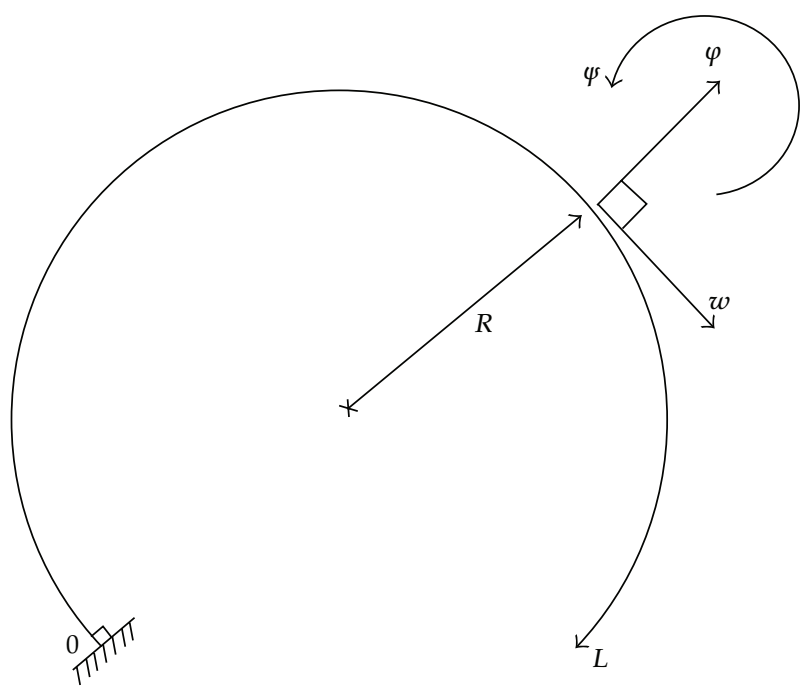

Figure 1: The circular arch.

where $\gamma_{1}, \gamma_{2}$ and $\gamma_{3}$ are positive constants. The initial conditions are

$\varphi(\cdot, 0)=\varphi_{0}, \quad \varphi_{t}(\cdot, 0)=\varphi_{1}, \quad \psi(\cdot, 0)=\psi_{0}, \quad \psi_{t}(\cdot, 0)=\psi_{1}, \quad w(\cdot, 0)=w_{0}, \quad w_{t}(\cdot, 0)=w_{1} \quad$ in $] 0, L[$,

and we assume the Dirichlet boundary conditions

$$
\varphi(0, t)=\varphi(L, t)=\psi(0, t)=\psi(L, t)=w(0, t)=w(L, t)=0 \quad \text { in }] 0, T[.
$$

Remark 1.1. If $R \rightarrow \infty$, then $l \rightarrow 0$, and this model reduces to the well-known Timoshenko beam equations (see [1] for details).

Related to the objectives of this paper there are few results in the literature. Recently, Liu and Rao [8] studied the asymptotic behavior of the circular arch in the context of linear thermoelasticity and they proved that the exponential decay occurs if and only if the speed of wave propagation that occur in the model are equal.

Our main result is to prove that the Bresse system is exponentially stable in the presence of feedback laws and in the sequence we evidence our result by using the finite difference method of locking-free nature. This result was not considered for circular arch problem.

The paper is organized as follows. In Section 2 we establish the existence, regularity, and uniqueness of global solutions of (1.4)-(1.8). We use the semigroup techniques. In Section 3 we study the exponential decay of the strong solutions to system (1.4)-(1.8). We show the exponential decay of the solution by using a method developed by Liu and Zheng [9] and their collaborators in past years. Finally, in Section 4 some numerical aspects were considered. We use a particular discretization in finite difference method that is locking free (see $[10,11])$ and our objective in this case is only to verify the numerical exponential decay for system (1.4)-(1.8). Questions about numerical analysis of numerical exponential decay 
as well the criterion stability for explicit time method in finite difference requires a more elaborate analysis.

\section{The Semigroup Setting}

In this section we will study the existence and uniqueness of strong and global solutions for the system (1.4)-(1.8) using the semigroup techniques. To give an accurate formulation of the evolution problem we are introducing the product Hilbert space

$$
\mathscr{H}:=H_{0}^{1}(0, L) \times L^{2}(0, L) \times H_{0}^{1}(0, L) \times L^{2}(0, L) \times H_{0}^{1}(0, L) \times L^{2}(0, L)
$$

with norm given by

$$
\begin{aligned}
\|U\|_{\mathscr{d}}^{2} & =\left\|(\varphi, \tilde{\varphi}, \psi, \tilde{\psi}, w, \tilde{w})^{T}\right\|_{\mathscr{d}}^{2} \\
& \equiv \int_{0}^{L} \rho_{1}|\tilde{\varphi}|^{2}+\rho_{2}|\tilde{\psi}|^{2}+\rho_{1}|\tilde{w}|^{2}+b\left|\psi_{x}\right|^{2}+\left.k\left|\varphi_{x}+\psi+\right| w\right|^{2}+k_{0}\left|w_{x}-l \varphi\right|^{2} d x .
\end{aligned}
$$

Let $U=\left(\varphi, \varphi_{t}, \psi, \psi_{t}, w, w_{t}\right)^{T}$, and we define the operator $\mathcal{A}: D(\mathcal{A}) \subset \mathscr{d} \rightarrow \mathscr{\ell}$ given by

$$
\mathcal{A}=\left(\begin{array}{cccccc}
0 & 1 & 0 & 0 & 0 & 0 \\
\frac{k}{\rho_{1} \partial_{x}^{2}}-\frac{k_{0} l^{2} I}{\rho_{1}} & -\frac{\gamma_{1}}{\rho_{1} I} & \frac{k}{\rho_{1} \partial_{x}} & 0 & \frac{\left(k+k_{0}\right) l}{\rho_{1} \partial_{x}} & 0 \\
0 & 0 & 0 & 1 & 0 & 0 \\
-\frac{k}{\rho_{2} \partial_{x}} & 0 & \frac{b}{\rho_{2} \partial_{x}^{2}}-\frac{k}{\rho_{2} I} & -\frac{\gamma_{2}}{\rho_{2} I} & -\frac{k l}{\rho_{2} I} & 0 \\
0 & 0 & 0 & 0 & 0 & 1 \\
-\frac{\left(k_{0}+k\right) l}{\rho_{1} \partial_{x}} & 0 & \frac{l k I}{\rho_{1}} & 0 & \frac{k_{0}}{\rho_{1} \partial_{x}^{2}}-\frac{l^{2} k}{\rho_{1} I} & -\frac{\gamma_{3}}{\rho_{1} I}
\end{array}\right)
$$

with domain

$$
D(\mathcal{A}):=\left(H_{0}^{1}(0, L) \cap H^{2}(0, L) \times H_{0}^{1}(0, L)\right)^{3} .
$$

Therefore, system (1.4)-(1.8) is equivalent to

$$
\begin{gathered}
U_{t}=\mathscr{A U}, \\
U(0)=U_{0},
\end{gathered}
$$


where $U_{0}:=\left(\varphi_{0}, \varphi_{1}, \psi_{0}, \psi_{1}, w_{0}, w_{1}\right)^{T}$. It is not difficult to see that $\mathbb{A}$ is a dissipative operator in the phase space $\mathscr{H}$. More precisely we have

$$
\operatorname{Re}(\mathcal{A} U, U)_{\mathscr{H}}=-\int_{0}^{L}\left[\frac{\gamma_{1}}{\rho_{1}}\left|\varphi_{t}\right|^{2}+\frac{\gamma_{2}}{\rho_{2}}\left|\varphi_{t}\right|^{2}+\frac{\gamma_{3}}{\rho_{1}}\left|w_{t}\right|^{2}\right] d x \leq 0
$$

Under the above notation we can establish the following theorem.

Theorem 2.1. The operator $A$ is the infinitesimal generator of $C_{0}$-semigroup $S(t)$ of contraction in th.

Proof. Since $\Phi(\mathcal{A})$ is dense in $\mathscr{t}$ and $\mathscr{A}$ is a dissipative operator, to prove Theorem 2.1 , it is sufficient to prove that 0 belongs to the resolvent set of $\mathcal{A}$, that is, $0 \in \rho(\mathcal{A})$ (see [12, Theorem 4.3]). Let us take $F=\left(f_{1}, f_{2}, f_{3}, f_{4}, f_{5}, f_{6}\right) \in \mathscr{d}$, then there is an only $U \in \mathscr{d}$ such that

$$
A U=F
$$

It easily follows from (2.7) or the standard result on the linear elliptic equations that (2.7) has a unique solution $(\varphi, \psi, w) \in\left(H_{0}^{1}(\Omega) \cap H^{2}(\Omega)\right)^{3}$. Therefore, $0 \in \rho(\mathcal{A})$ and $\left(\varphi, \varphi_{t}, \psi, \psi_{t}, w, w_{t}\right) \in$ $\Phi(\mathcal{A})$. The proof is now complete.

\section{Exponential Decay}

In this section we will prove that the semigroup $S(t)$ on $\mathscr{t}$ is exponentially stable. Here we will use necessary and sufficient conditions for $C_{0}$-semigroups being exponentially stable in a Hilbert space. This result was obtained by Gearhart [13] and Huang [14], independently (see also [15]).

Theorem 3.1. Let $S(t)=e^{A t}$ be a $C_{0}$-semigroup of contractions on Hilbert space $\mathcal{H}$. Then $S(t)$ is exponentially stable if and only if

$$
\begin{gathered}
\rho(\mathcal{A}) \supseteq\{i \lambda: \lambda \in \mathbb{R}\} \equiv i \mathbb{R}, \\
\lim _{|\lambda| \rightarrow \infty}\left\|(i \lambda I-\mathcal{A})^{-1}\right\|_{\mathcal{L}(\mathscr{l})}<\infty
\end{gathered}
$$

hold, where $\rho(\mathcal{A})$ is the resolvent set of $\mathcal{A}$.

Theorem 3.2. The $C_{0}$-semigroup of contractions $\left(e^{A t}\right), t>0$, generated by $\mathcal{A}$, is exponentially stable.

Proof. To prove the exponential stability of $\left(e^{\mathscr{A t}}\right)$, it remains to verify the properties (3.1) and (3.2) of Theorem 3.1. First we prove that

$$
\rho(\mathcal{A}) \supseteq\{i \lambda: \lambda \in \mathbb{R}\} .
$$


Suppose that the conclusion of (3.1) is not true. Then there is a $\beta \in \mathbb{R}$ such that, $\beta \neq 0$, i $\beta \in$ $\sigma(A)$ (spectrum of $\mathcal{A}$ ) and since $\mathcal{A}^{-1}$ is compact, i $\beta$ must be an eigenvalue of $\mathcal{A}$. Let $U=$ $\left(\varphi, \varphi_{t}, \psi, \psi_{t}, w, w_{t}\right)^{T}$ be with $\|U\|_{\mathscr{\ell}}=1$, such that $\mathcal{A} U=i \beta U$. Using the definition of $\mathcal{A}$ it follows that $A U=i \beta U$ if and only if

$$
\begin{gathered}
\frac{k}{\rho_{1} \varphi_{x x}}-\frac{k_{0}}{\rho_{1} l^{2} \varphi}+\frac{k}{\rho_{1} \psi_{x}}+\frac{\left(k+k_{0}\right)}{\rho_{1} l w_{x}}+\beta^{2} \varphi=\frac{i \gamma_{1}}{\rho_{1} \beta \varphi^{\prime}} \\
\frac{b}{\rho_{1} \psi_{x x}}-\frac{k}{\rho_{2} \psi}-\frac{k}{\rho_{2} \varphi_{x}}-\frac{k}{\rho_{2} l w}+\beta^{2} \psi=\frac{i \gamma_{2}}{\rho_{2} \beta \psi} \\
\frac{k_{0}}{\rho_{1} w_{x x}}-\frac{k}{\rho_{1} l^{2} w}-\frac{\left(k+k_{0}\right)}{\rho_{1} l \varphi_{x}}+\frac{k}{\rho_{1} l \psi}+\beta^{2} w=\frac{i \gamma_{3}}{\rho_{1} w}
\end{gathered}
$$

Multiplying equations (3.4) (3.5), and (3.6) by $\varphi, \psi$ and $w$, respectively, and performing integration by parts on $] 0, L[$, we arrive at

$$
\begin{aligned}
& \gamma_{1} \beta \int_{0}^{L} \varphi^{2} d x=0, \\
& \gamma_{2} \beta \int_{0}^{L} \psi^{2} d x=0, \\
& \gamma_{3} \beta \int_{0}^{L} w^{2} d x=0,
\end{aligned}
$$

which implies that $\varphi=0, \psi=0, w=0$ and consequently

$$
\int_{0}^{L} b\left|\psi_{x}\right|^{2}+k\left|\varphi_{x}+\psi+l w\right|^{2}+k_{0}\left|w_{x}-l \varphi\right|^{2} d x=0
$$

from where we can conclude that $\|U\|_{\mathscr{H}}=0$, which contradicts $\|U\|_{\mathscr{H}}=1$. This completes the proof of (3.1).

We now prove (3.2) by a contradiction argument again. Suppose that (3.2) is not true. Then there are a sequence $V_{n} \in \mathscr{H}$ and a sequence $\beta_{n} \in \mathbb{R}$ such that

$$
n\left\|V_{n}\right\|_{\mathscr{H}} \leq\left\|\left(I \beta_{n} I-\mathcal{A}\right)^{-1} V_{n}\right\|_{\mathscr{\ell ^ { \prime }}} \quad \forall n>0 .
$$

From $i \beta_{n} \in \rho(\mathcal{A})$ we have that there exists a unique sequence $U_{n} \in \Phi(\mathcal{A})$ such that

$$
i \beta_{n} U_{n}-\mathscr{A} U_{n}=V_{n}, \quad\left\|U_{n}\right\|_{\mathscr{L}}=1 .
$$

Denoting by $F_{n}=i \beta_{n} U_{n}-\mathcal{A} U_{n}$ it follows that

$$
\left\|F_{n}\right\|_{\mathscr{d}} \leq \frac{1}{n} \longrightarrow 0, \quad \text { as } n \longrightarrow \infty
$$


Journal of Applied Mathematics

Let us denote by

$$
\begin{gathered}
U_{n}=\left(\varphi^{n}, \tilde{\varphi}^{n}, \psi^{n}, \tilde{\psi}^{n}, w^{n}, \tilde{w}^{n}\right)^{T} \\
F_{n}=\left(f_{1}^{n}, f_{2}^{n}, f_{3}^{n}, f_{4}^{n}, f_{5}^{n}, f_{6}^{n}\right)^{T} .
\end{gathered}
$$

From $F_{n}=i \beta_{n} U_{n}-\mathcal{A} U_{n}$ we have the following equations in $L^{2}(0, L)$ :

$$
\begin{gathered}
i \beta_{n} \varphi^{n}-\tilde{\varphi}^{n}=f_{1}^{n}, \\
i \rho_{1} \beta_{n} \widetilde{\varphi}^{n}-k \varphi_{x x}^{n}+k_{0} l^{2} \varphi^{n}+\gamma_{1} \tilde{\varphi}^{n}-k \psi_{x}^{n}-\left(k+k_{0}\right) l w_{x}^{n}=f_{2}^{n}, \\
i \beta_{n} \psi^{n}-\tilde{\psi}^{n}=f_{3}^{n}, \\
i \rho_{2} \beta_{n} \tilde{\psi}^{n}-b \psi_{x x}^{n}+k \psi^{n}+k \varphi_{x}^{n}+\gamma_{2} \tilde{\psi}^{n}+k l w^{n}=f_{4}^{n}, \\
i \beta_{n} w^{n}-\widetilde{w}^{n}=f_{5}^{n}, \\
i \rho_{1} \beta_{n} \tilde{w}-k_{0} w_{x x}^{n}+k l^{2} w^{n}+\gamma_{3} \tilde{w}^{n}+\left(k+k_{0}\right) l \varphi_{x}^{n}+l k \psi^{n}=f_{6}^{n} .
\end{gathered}
$$

Taking the inner product of $\left(i \beta_{n} I-\mathscr{A}\right) U^{n}$ with $U^{n}$ in $\mathscr{H}$ and then taking the real part, we obtain

$$
\operatorname{Re}\left(\left(i \beta_{n} I-\mathcal{A}\right) U^{n}, U^{n}\right)_{\mathscr{H}}=-\int_{0}^{L}\left[\frac{\gamma_{1}}{\rho_{1}}\left|\tilde{\varphi}^{n}\right|^{2}+\frac{\gamma_{2}}{\rho_{2}}\left|\tilde{\psi}^{n}\right|^{2}+\frac{\gamma_{3}}{\rho_{1}}\left|\tilde{w}^{n}\right|^{2}\right] d x \rightarrow 0
$$

from where we can conclude that

$$
\begin{aligned}
& \tilde{\varphi}^{n} \longrightarrow 0 \quad \text { in } L^{2}(0, L), \\
& \tilde{\varphi}^{n} \longrightarrow 0 \quad \text { in } L^{2}(0, L), \\
& \widetilde{w}^{n} \longrightarrow 0 \quad \text { in } L^{2}(0, L) .
\end{aligned}
$$

Considering the equation

$$
\beta_{n}\left\|U^{n}\right\|^{2}-i\left(\mathscr{A} U^{n}, U^{n}\right)_{\mathscr{d}}=i\left(F^{n}, U^{n}\right)_{\mathscr{H}}
$$

then from (2.6)

$$
\beta_{n}\left\|U^{n}\right\|^{2}+i \int_{0}^{L}\left[\frac{\gamma_{1}}{\rho_{1}}\left|\tilde{\varphi}^{n}\right|^{2}+\frac{\gamma_{2}}{\rho_{2}}\left|\tilde{\psi}^{n}\right|^{2}+\frac{\gamma_{3}}{\rho_{1}}\left|\tilde{w}^{n}\right|^{2}\right] d x=i\left(F^{n}, U^{n}\right)_{\mathscr{d}}
$$

which implies that $\beta_{n}\left\|U^{n}\right\|^{2} \rightarrow 0$, and then

$$
\beta_{n}\left|\tilde{\varphi}^{n}\right|^{2} \longrightarrow 0, \quad \beta_{n}\left|\tilde{\psi}^{n}\right|^{2} d x \longrightarrow 0, \quad \beta_{n}\left|\tilde{w}^{n}\right|^{2} \longrightarrow 0 .
$$


From

$$
\tilde{\varphi}^{n} \longrightarrow 0, \quad \tilde{\psi}^{n} \longrightarrow 0, \quad \tilde{w}^{n} \longrightarrow 0
$$

we can conclude that

$$
\beta_{n} \tilde{\varphi}^{n} \longrightarrow 0, \quad \beta_{n} \tilde{\psi}^{n} \longrightarrow 0, \quad \beta_{n} \tilde{w}^{n} \longrightarrow 0
$$

From equations (3.14), (3.16) and (3.18) we obtain, after some calculations,

$$
\int_{0}^{L} b\left|\psi_{x}^{n}\right|^{2}+k\left|\varphi_{x}^{n}+\psi^{n}+l w^{n}\right|^{2}+k_{0}\left|w_{x}^{n}-l \varphi^{n}\right|^{2} d x \longrightarrow 0
$$

In summary, we have proved that $\left\|U^{n}\right\|_{\mathscr{H}} \rightarrow 0$ which contradicts $\left\|U^{n}\right\|_{\mathscr{\ell}}=1$. Thus, the proof is complete.

\section{Unlocking for Spatial Finite Difference Scheme}

For numerical verification of the exponential decay of system (1.4)-(1.8) we use the total discretization in finite difference method. With respect to numerical schemes in this case, some aspects should be taking into account. The first one concerns to numerical phenomenon known as shear locking, which affects some numerical models applied to vibration problems in structures as shell, plates and beams. For the locking phenomenon (see [4-7]) for ample discussion for plane beams. For fast discussion about this numerical problem, we considerer the case of plane beams described by theory of Timoshenko and given by the following equations:

$$
\begin{gathered}
\rho_{1} \varphi_{t t}-\kappa\left(\varphi_{x}+\psi\right)_{x}=0, \\
\rho_{2} \psi_{t t}-b \psi_{x x}+\kappa\left(\varphi_{x}+\psi\right)=0 .
\end{gathered}
$$

are resulting of the equations (1.4)-(1.5) for $l \rightarrow 0$.

By using the finite element method standard with linear shape functions, the rigidity coefficient $b=E I$ is modified for

$$
\mathbf{b}^{*}=b\left(1+\frac{\kappa}{12 b} h^{2}\right)=E I\left[1+\frac{k^{\prime} G}{E}\left(\frac{h}{\epsilon}\right)^{2}\right]
$$

where $b=E I, \kappa=k G A, A=a \epsilon, I=a \epsilon^{3} / 12$ considering a rectangular geometry with width a and thickness $\epsilon$. In particular, for plane beams must be have $\epsilon<h$ where $h$ is the spatial division (see ([10,11] for details). Consequently, $\mathbf{b}^{*}>b$ and clearly this value for rigidity 


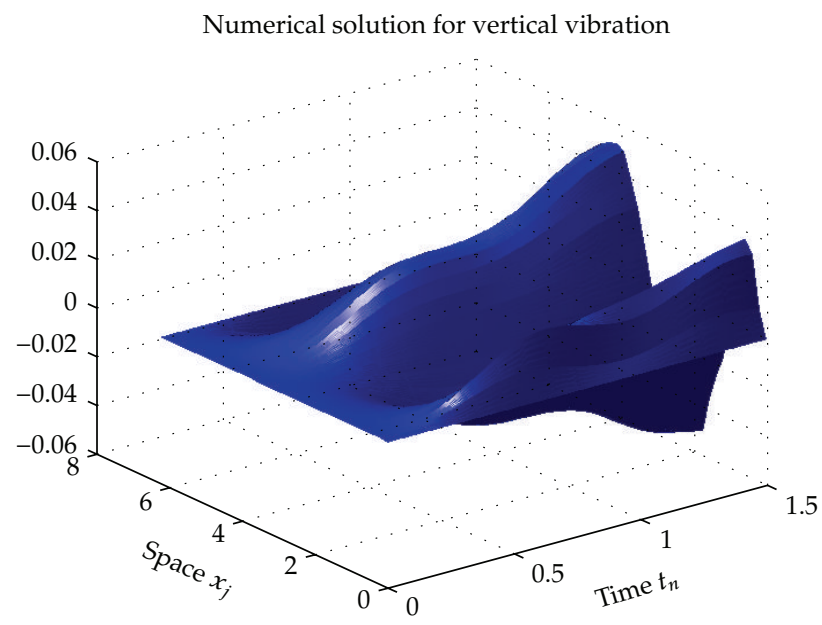

Figure 2: Conservative case, $\mu=3$.

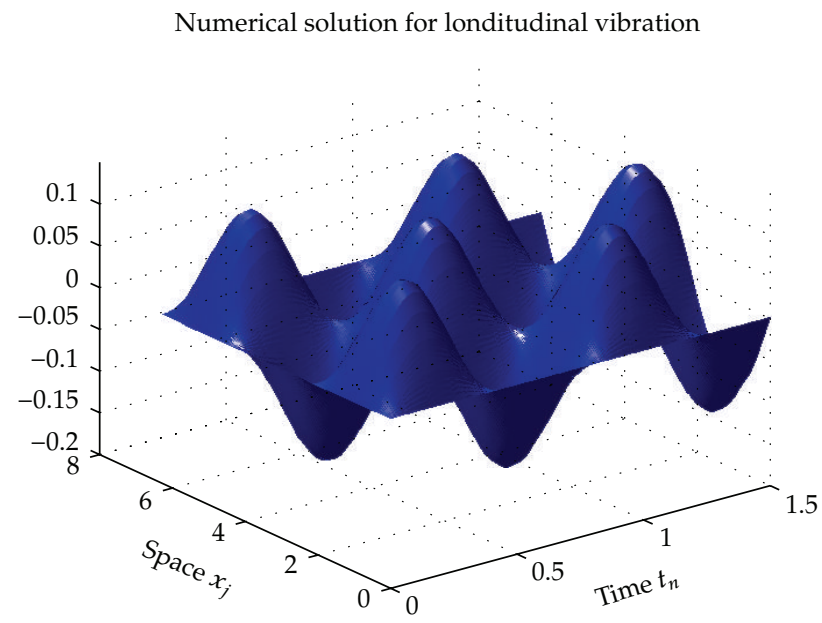

Figure 3: Conservative case, $\mu=3$.

coefficient does not correspond to the real case for $h$ fixed. In reality, with $\mathbf{b}^{*}$ the equation for angle rotation in (4.2) is rewrite as

$$
\rho_{2} \psi_{t t}-b \psi_{x x}-\frac{\kappa}{12} h^{2} \psi_{x x}+\kappa\left(\varphi_{x}+\psi\right)=0
$$

and the energy respective is

$$
E(t)=\frac{1}{2} \int_{0}^{L}\left[\rho_{1}\left|\varphi_{t}\right|^{2}+\rho_{2}\left|\psi_{t}\right|^{2}\right] d x+\frac{1}{2} \int_{0}^{L}\left[\kappa\left|\varphi_{x}+\psi\right|^{2}+\mathbf{b}^{*}\left|\psi_{x}\right|^{2}\right] d x
$$




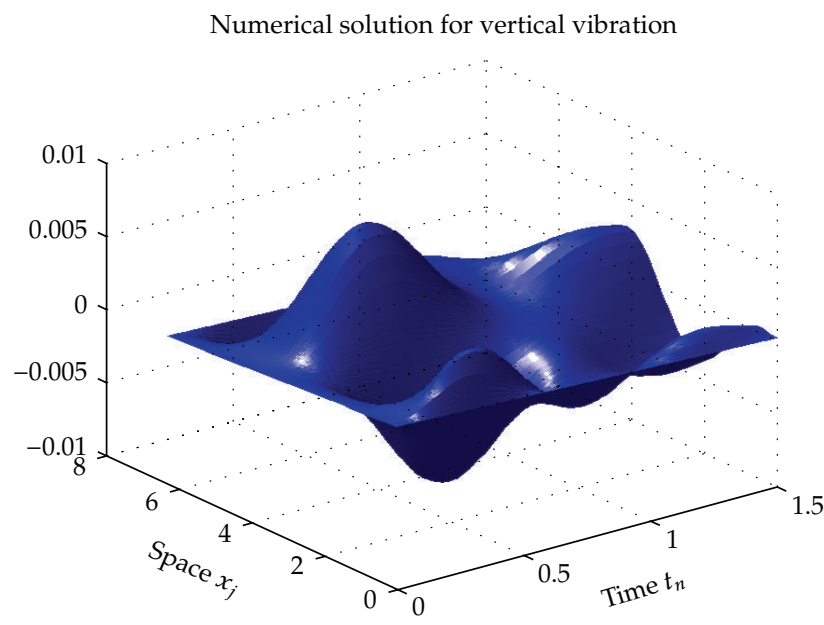

Figure 4: Total dissipation, $\mu=3$.

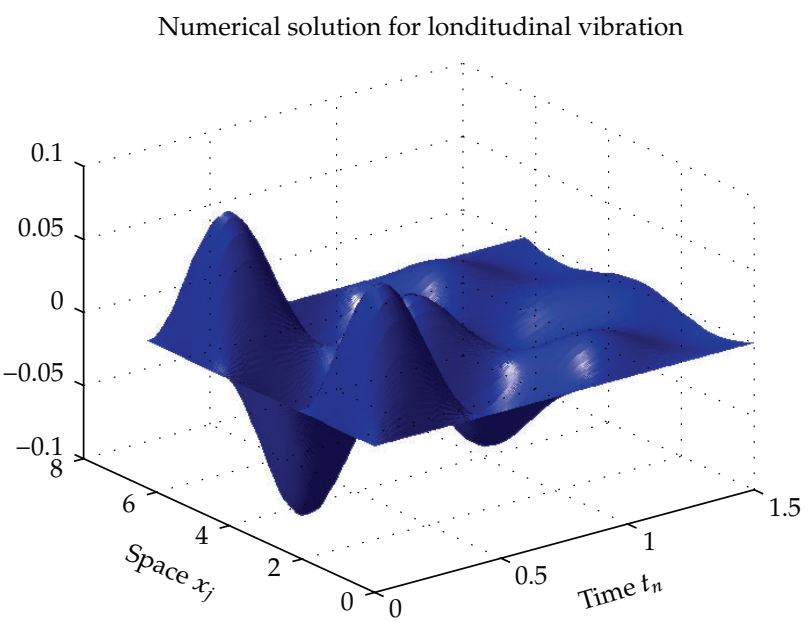

Figure 5: Total dissipation, $\mu=3$.

and obviously this energy is different of the real case with $\mathbf{b}^{*}=b$. In general the numerical schemes in finite element method or finite difference method are locking free/over-estimation if not exist any additional term about the coefficients of the system, because the shear locking problem is basically a numerical anomaly characterized by a over-estimation about rigidity coefficients and dependent of parameter $h$.

To avoid this numerical anomaly the finite element method can be used, however, special care must be taking in to account for choose of basis functions [6, 7]. Naturally, this shear locking/over-estimation can be to affect equations (1.4)-(1.6). See the studies by Loula et al. in [16-18] for numerical treatment in finite element for circular arch problem.

In our case, we use the total discretization in finite difference method and to avoid shear locking/over-estimation we make a particular discretization for the functions of zero derivative such as $\kappa_{0} l^{2} \varphi, \kappa \psi$ and $\kappa l w$ for a numerical operator of second order in relation to $\Delta x$. 


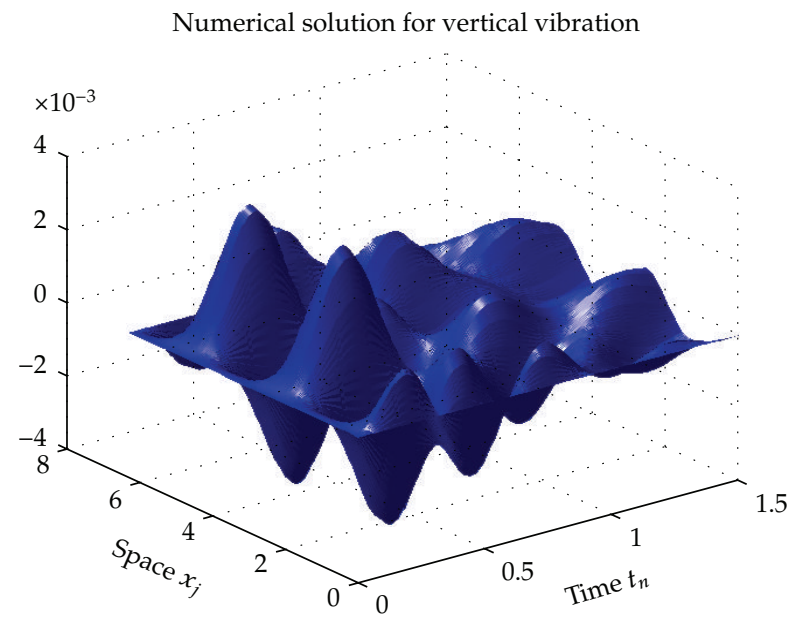

Figure 6: Total dissipation, $\mu=5$.

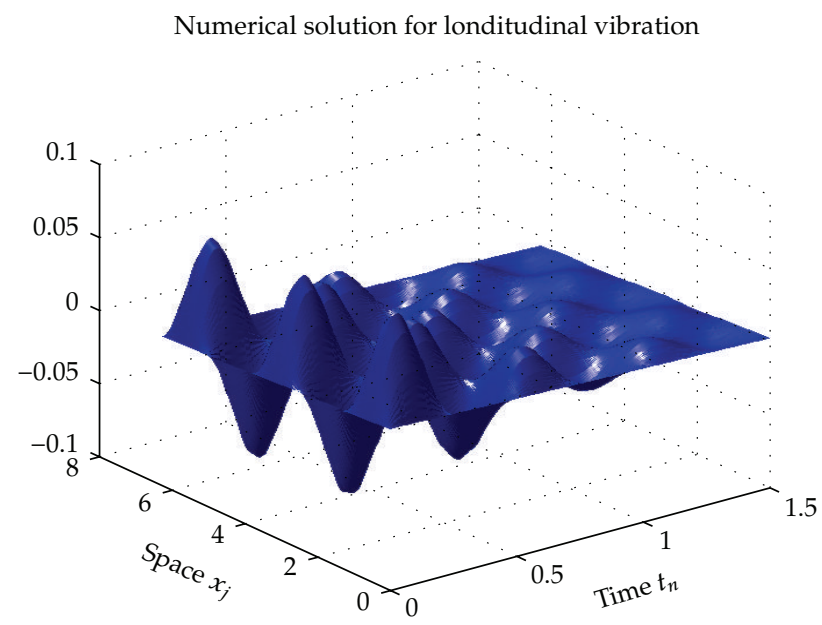

Figure 7: Total dissipation, $\mu=5$.

For our purposes, we use the space-time explicit method applied to equations (1.4)(1.6) and we define $\Delta x=L /(J+1), \Delta t=T /(N+1)$ for $J, N \in \mathbb{N}$ and nets

$$
\begin{aligned}
& x_{0}=0<x_{1}=\Delta x<\cdots<x_{J}=J \Delta x<x_{J+1}=L, \\
& t_{0}=0<t_{1}=\Delta t<\cdots<t_{N}=N \Delta t<t_{N+1}=T,
\end{aligned}
$$

where $x_{j}=j \Delta x$ and $t_{n}=n \Delta t$ for $j=0,1,2, \ldots, J+1$ and $n=0,1,2, \ldots, N+1$. The numerical 


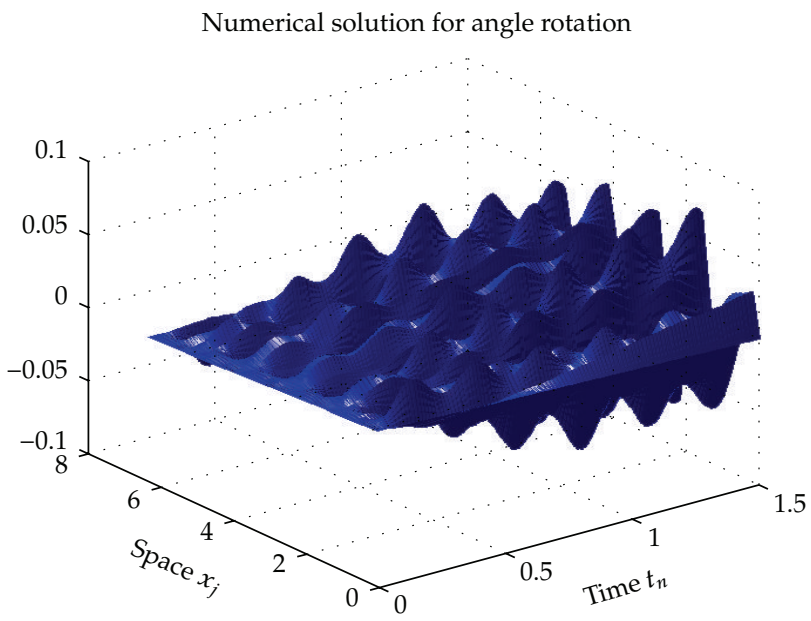

Figure 8: Conservative case, $\mu=10$.

scheme consists in to find $\varphi_{j}^{n+1}, \psi_{j}^{n+1}$ and $w_{j}^{n+1}$ such that

$\rho_{1} \bar{\partial}_{t} \partial_{t} \varphi_{j}^{n}=\kappa \bar{\partial}_{x} \partial_{x} \varphi_{j}^{n}+\kappa \frac{\partial_{x}+\bar{\partial}_{x}}{2} \psi_{j}^{n}+\bar{\kappa} \frac{\partial_{x}+\bar{\partial}_{x}}{2} w_{j}^{n}-\frac{\kappa_{0} l^{2}}{2}\left(\varphi_{j-1 / 2}^{n}+\varphi_{j+1 / 2}^{n}\right)-\gamma_{1} \frac{\partial_{t}+\bar{\partial}_{t}}{2} \varphi_{j}^{n}$,

$\rho_{2} \bar{\partial}_{t} \partial_{t} \psi_{j}^{n}=b \bar{\partial}_{x} \partial_{x} \psi_{j}^{n}-\kappa \frac{\partial_{x}+\bar{\partial}_{x}}{2} \varphi_{j}^{n}-\frac{\kappa}{2}\left(\psi_{j-1 / 2}^{n}+\psi_{j+1 / 2}^{n}\right)-\frac{\kappa l}{2}\left(w_{j-1 / 2}^{n}+w_{j+1 / 2}^{n}\right)-\gamma_{2} \frac{\partial_{t}+\bar{\partial}_{t}}{2} \psi_{j}^{n}$,

$\rho_{1} \bar{\partial}_{t} \partial_{t} w_{j}^{n}=\kappa_{0} \bar{\partial}_{x} \partial_{x} w_{j}^{n}-\bar{\kappa} \frac{\partial_{x}+\bar{\partial}_{x}}{2} \varphi_{j}^{n}-\frac{\kappa l}{2}\left(\psi_{j-1 / 2}^{n}+\psi_{j+1 / 2}^{n}\right)-\frac{\kappa l^{2}}{2}\left(w_{j-1 / 2}^{n}+w_{j+1 / 2}^{n}\right)-\gamma_{3} \frac{\partial_{t}+\bar{\partial}_{t}}{2} w_{j}^{n}$

for $j=1,2, \ldots, J, n=1,2, \ldots, N$, with the following numerical operators of second order for a function $u(x, t)$ :

$$
\begin{array}{cc}
\frac{\partial_{x}+\bar{\partial}_{x}}{2} u_{j}^{n}=\frac{u_{j+1}^{n}-u_{j-1}^{n}}{2 \Delta x}, & \frac{\partial_{t}+\bar{\partial}_{t}}{2} u_{j}^{n}=\frac{u_{j}^{n+1}-u_{j}^{n-1}}{2 \Delta t}, \\
\bar{\partial}_{x} \partial_{x} u_{j}^{n}=\frac{u_{j+1}^{n}-2 u_{j}^{n}+u_{j-1}^{n}}{\Delta x^{2}}, & \bar{\partial}_{t} \partial_{t} u_{j}^{n}=\frac{u_{j}^{n+1}-2 u_{j}^{n}+u_{j}^{n-1}}{\Delta t^{2}} .
\end{array}
$$

For $u_{j-1 / 2}^{n}$ and $u_{j+1 / 2}^{n}$ we denote the average of $u\left(x_{j}, t_{n}\right)$ on the points $\left(x_{j-1}, t_{n}\right),\left(x_{j}, t_{n}\right)$ and $\left(x_{j+1}, t_{n}\right),\left(x_{j}, t_{n}\right)$, respectively. This approximation avoid any over-estimation about the coefficients of equations (1.4)-(1.6). Then, we have,

$$
\frac{u_{j-1 / 2}^{n}+u_{j+1 / 2}^{n}}{2}:=\frac{u_{j+1}^{n}+2 u_{j}^{n}+u_{j-1}^{n}}{4} .
$$




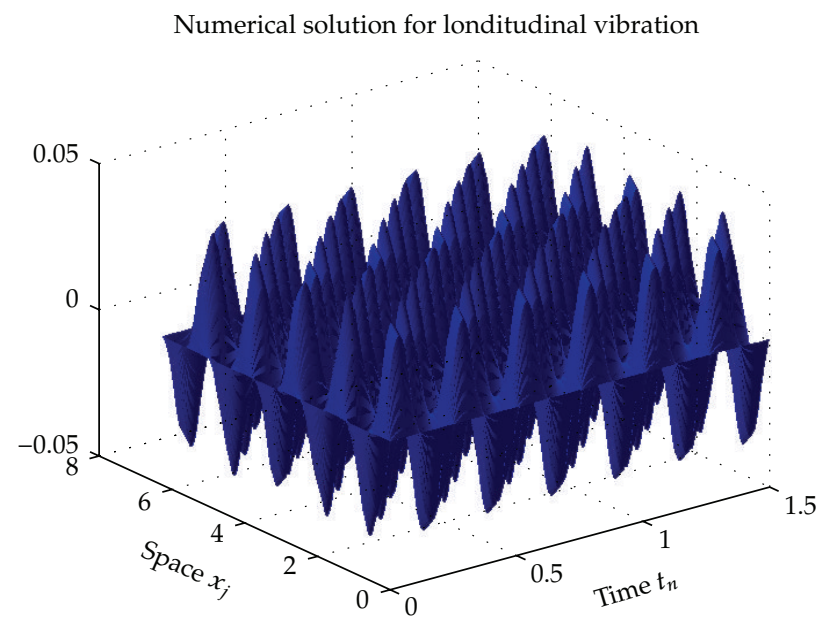

Figure 9: Conservative case, $\mu=10$.

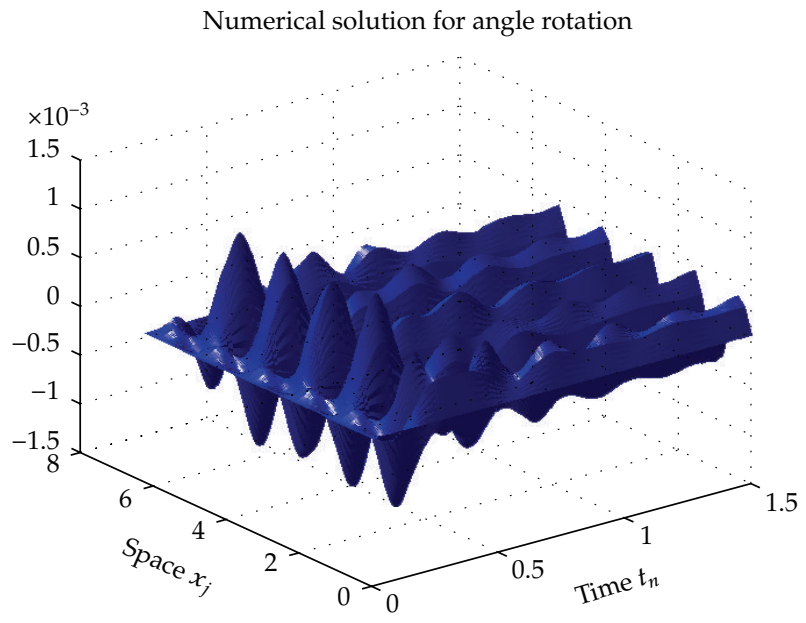

Figure 10: Total dissipation case, $\mu=10$.

The boundary conditions in the numerical context are given by

$$
\varphi_{0}^{n}=\varphi_{J+1}^{n}=0, \quad \psi_{0}^{n}=\psi_{J+1}^{n}=0, \quad w_{0}^{n}=w_{J+1}^{n}=0, \quad \forall n=1,2, \ldots, N,
$$

and the discretizations to initial conditions are given by

$$
\begin{aligned}
& \varphi_{j}^{0}=\varphi\left(x_{j}, 0\right), \quad \varphi_{j}^{1}=\varphi_{j}^{-1}+2 \Delta t \varphi_{t}\left(x_{j}, 0\right), \quad \forall j=1,2, \ldots, J, \\
& \psi_{j}^{0}=\psi\left(x_{j}, 0\right), \quad \psi_{j}^{1}=\psi_{j}^{-1}+2 \Delta t \psi_{t}\left(x_{j}, 0\right), \quad \forall j=1,2, \ldots, J, \\
& w_{j}^{0}=w\left(x_{j}, 0\right), \quad w_{j}^{1}=w_{j}^{-1}+2 \Delta t w_{t}\left(x_{j}, 0\right), \quad \forall j=1,2, \ldots, J .
\end{aligned}
$$




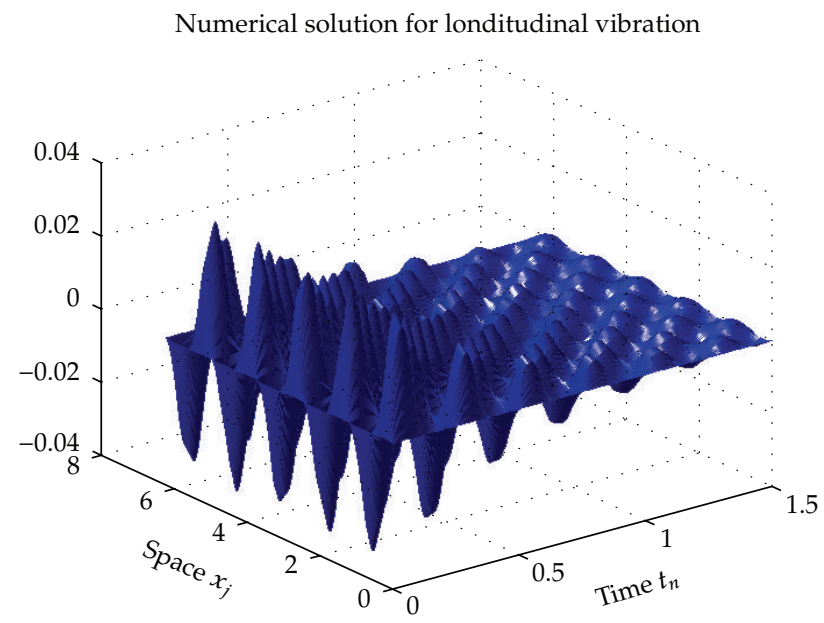

Figure 11: Total dissipation, $\mu=10$.

The numerical energy associated for (4.7)-(4.13) is given by

$$
\begin{aligned}
E^{n}:=\frac{\Delta x}{2} \sum_{j=0}^{J} & {\left[\rho_{1}\left(\frac{\varphi_{j}^{n+1}-\varphi_{j}^{n}}{\Delta t}\right)^{2}+\rho_{2}\left(\frac{\psi_{j}^{n+1}-\psi_{j}^{n}}{\Delta t}\right)^{2}+\rho_{1}\left(\frac{w_{j}^{n+1}-w_{j}^{n}}{\Delta t}\right)^{2}\right.} \\
& +b\left(\frac{\psi_{j+1}^{n+1}-\psi_{j}^{n+1}}{\Delta x}\right)\left(\frac{\psi_{j+1}^{n}-\psi_{j}^{n}}{\Delta x}\right) \\
& +\kappa_{0}\left(\frac{w_{j+1}^{n+1}-w_{j}^{n+1}}{\Delta x}-l \frac{\varphi_{j+1}^{n+1}+\varphi_{j}^{n+1}}{2}\right)\left(\frac{w_{j+1}^{n}-w_{j}^{n}}{\Delta x}-l \frac{\varphi_{j+1}^{n}+\varphi_{j}^{n}}{2}\right) \\
& \left.+\kappa\left(\frac{\varphi_{j+1}^{n}-\varphi_{j}^{n}}{\Delta x}+\frac{\psi_{j+1}^{n}+\psi_{j}^{n}}{2}+l \frac{w_{j+1}^{n}+w_{j}^{n}}{2}\right)\right] .
\end{aligned}
$$

Equations (4.7)-(4.9), in fact, are locking free because the numerical energy (4.14) is compatible with the continuous energy in (2.2), because the coefficients $b, \kappa_{0}$ and $\kappa$ are exactly those in (2.2), without any dependence with $h$. To verify this, we have the following proposition.

Proposition 4.1. For all $\Delta t, \Delta x \in(0, L)$ the energy (4.14) of solutions of the discrete equations (4.7)-(4.9), with initial conditions (4.13) and any boundary conditions (4.12) is such that

$$
E^{n} \leq E^{0}, \quad \forall n=1,2, \ldots, N .
$$

Proof. The proof is more extensive and we have omitted it here. For a idea of the proof, we use the multiplicative techniques such as performed in $[19,20]$, that is we multiply the equations 


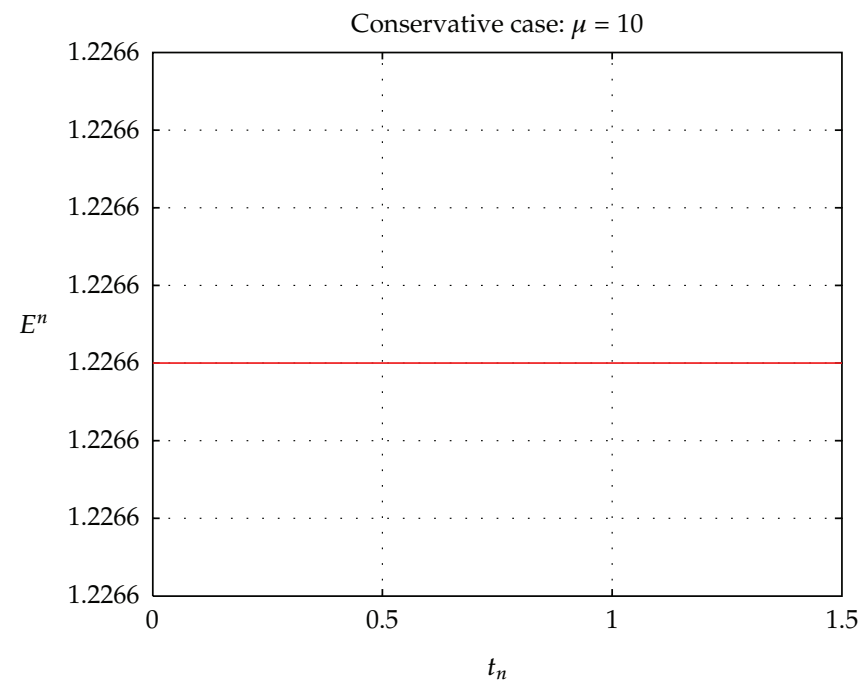

Figure 12: Conservative case.

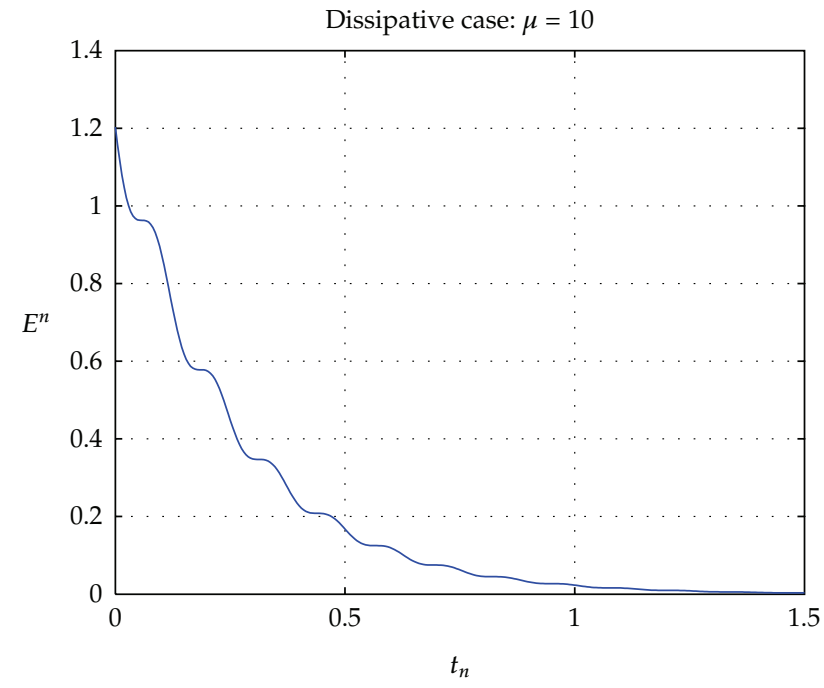

Figure 13: Dissipative case.

(4.7)-(4.9) by $(1 / 2)\left(\varphi_{j}^{n+1}-\varphi_{j}^{n-1}\right),(1 / 2)\left(\psi_{j}^{n+1}-\psi_{j}^{n-1}\right)$ and $(1 / 2)\left(w_{j}^{n+1}-w_{j}^{n-1}\right)$, respectively, and after we applied the discrete sum for $j=1, \ldots, J$. Then after some properly simplifications and taking in to account the boundary conditions given in (4.12) we get (4.14).

It is clear that discrete equations (4.7)-(4.9) are all consistent with $\mathcal{O}\left(\Delta x^{2}, \Delta t^{2}\right)$ for truncation error. Then, by Lax equivalence theorem the equations (4.7)-(4.9) are convergent if, and only if, they are stable. But another numerical limitation occurs for explicit time methods when applied to vibrations problem in elastic structures, with, say, respect to numerical stability. In particular, for explicit time method in finite difference applied to vibrations in Timoshenko beams, the prevailing numerical stability is given by $\Delta t \leq \epsilon / \sqrt{3} c_{s}$ 
where $c_{s}=\sqrt{k^{\prime} G / \rho}$. The limitation occurs when $\epsilon$ is small. The understanding and overcoming for this limitation was studied by Joseph P. Wright in [10,11]. Naturally, the same numerical problem affect (4.7)-(4.9), in function of the dependence of thickness $\epsilon$. Then, this problem should be the objective of study in another opportunity, and for our purpose of numerical verification to exponential decay, we consider $\epsilon$ fixed.

For numerical example, we consider $L=2 \pi \mathrm{m}$, thickness $\epsilon=0.025 \mathrm{~m}$, width $a=$ $0.0040 \mathrm{~m}, l=1 / 20, E=21 \times 10^{4} \mathrm{~N} / \mathrm{m}^{2}, \rho=7850 \mathrm{~kg} / \mathrm{m}^{3}, k^{\prime}=5 / 6, r=0.29$ (Poisson ratio), $G=E /(2+2 r)$ and the following initial conditions:

$$
\begin{gathered}
\varphi\left(x_{j}, 0\right)=\psi\left(x_{j}, 0\right)=w\left(x_{j}, 0\right)=0, \\
w_{t}\left(x_{j}, 0\right)=\sin \left(\frac{\mu \pi x_{j}}{L}\right), \quad \varphi_{t}\left(x_{j}, 0\right)=\psi_{t}\left(x_{j}, 0\right)=0, \quad \mu \in \mathbb{N} .
\end{gathered}
$$

First we reproduce the conservative case, $\gamma_{i}=0, i=1,2,3$ and for dissipative case, we consider $\gamma_{i}=\pi, i=1,2,3$. For the computational domain chosen 32 spatial points and 312 points in the time domain were given by $T=1.5$ seconds (see Figures $1,2,3,4,5,6$, and 7).

With Figures $8,9,10,11,12$, and 13 we illustrate the simulation results for vibration in angle rotation $\psi$ and the behavior of numerical energy in the cases conservative and dissipative. Of course the numerical energy (4.14) must be conservative in the absence of damping.

\section{Acknowledgments}

The first author acknowledges the support of the CNPq (306338/2008-4) and FAPESPA-PABrazil. The authors are thankful to the referees of this paper for the valuable suggestions which improved the paper.

\section{References}

[1] J. E. Lagnese, G. Leugering, and E. J. P. G. Schmidt, Modeling, Analysis and Control of Dynamic Elastic Multi-Link Structures, Systems \& Control: Foundations \& Applications, Birkhäuser, Boston, Mass, USA, 1994.

[2] J. E. Muñoz Rivera and R. Racke, "Mildly dissipative nonlinear Timoshenko systems-global existence and exponential stability," Journal of Mathematical Analysis and Applications, vol. 276, no. 1, pp. 248-278, 2002.

[3] J. E. Muñoz Rivera and R. Racke, "Global stability for damped Timoshenko systems," Discrete and Continuous Dynamical Systems. Series A, vol. 9, no. 6, pp. 1625-1639, 2003.

[4] A. F. D. Loula, T. J. R. Hughes, and L. P. Franca, "Petrov-Galerkin formulations of the Timoshenko beam problem," Computer Methods in Applied Mechanics and Engineering, vol. 63, no. 2, pp. 115-132, 1987.

[5] A. F. D. Loula, T. J. R. Hughes, L. P. Franca, and I. Miranda, "Mixed Petrov-Galerkin methods for the Timoshenko beam problem," Computer Methods in Applied Mechanics and Engineering, vol. 63, no. 2, pp. 133-154, 1987.

[6] G. Prathap and G. R. Bhashyam, "Reduced integration and the shear-flexible beam element," International Journal for Numerical Methods in Engineering, vol. 18, no. 2, pp. 195-210, 1982.

[7] T. J. R. Hughes, R. L. Taylor, and W. Kanoknukulchai, “A simple and efficient finite element method for plate bending," International Journal for Numerical Methods in Engineering, vol. 11, no. 10, pp. 1529 $1543,1977$.

[8] Z. Liu and B. Rao, "Energy decay rate of the thermoelastic Bresse system," Zeitschrift für Angewandte Mathematik und Physik, vol. 60, no. 1, pp. 54-69, 2009. 
[9] Z. Liu and S. Zheng, Semigroups Associated with Dissipative Systems, vol. 398 of Chapman E Hall/CRC Research Notes in Mathematics, Chapman \& Hall/CRC, Boca Raton, Fla, USA, 1999.

[10] J. P. Wright, "A mixed time integration method for Timoshenko and Mindlin type elements," Communications in Applied Numerical Methods, vol. 3, no. 3, pp. 181-185, 1987.

[11] J. P. Wright, "Numerical stability of a variable time step explicit method for Timoshenko and Mindlin type structures," Communications in Numerical Methods in Engineering, vol. 14, no. 2, pp. 81-86, 1998.

[12] A. Pazy, Semigroups of Linear Operators and Applications to Partial Differential Equations, vol. 44 of Applied Mathematical Sciences, Springer, New York, NY, USA, 1983.

[13] L. Gearhart, "Spectral theory for contraction semigroups on Hilbert space," Transactions of the American Mathematical Society, vol. 236, pp. 385-394, 1978.

[14] F. L. Huang, "Characteristic conditions for exponential stability of linear dynamical systems in Hilbert spaces," Annals of Differential Equations, vol. 1, no. 1, pp. 43-56, 1985.

[15] J. Prüss, "On the spectrum of $C_{0}$-semigroups," Transactions of the American Mathematical Society, vol. 284 , no. 2, pp. 847-857, 1984.

[16] A. F. D. Loula, L. P. Franca, T. J. R. Hughes, and I. Miranda, "Stability, convergence and accuracy of a new finite element method for the circular arch problem," Computer Methods in Applied Mechanics and Engineering, vol. 63, no. 3, pp. 281-303, 1987.

[17] T. J. R. Hughes, R. L. Taylor, and W. Kanoknukulchai, "A simple and efficient finite element for plate bending," International Journal for Numerical Methods in Engineering, vol. 11, no. 10, pp. 1529-1543, 1977.

[18] B. D. Reddy and M. B. Volpi, "Mixed finite element methods for the circular arch problem," Computer Methods in Applied Mechanics and Engineering, vol. 97, no. 1, pp. 125-145, 1992.

[19] M. Negreanu and E. Zuazua, "Uniform boundary controllability of a discrete 1-D wave equation," Systems \& Control Letters, vol. 48, no. 3-4, pp. 261-279, 2003.

[20] W. Strauss and L. Vazquez, "Numerical solution of a nonlinear Klein-Gordon equation," Journal of Computational Physics, vol. 28, no. 2, pp. 271-278, 1978. 


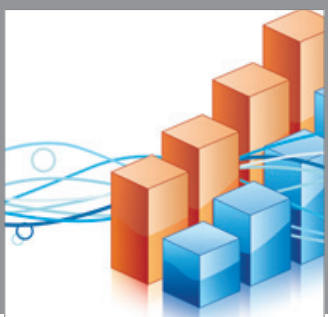

Advances in

Operations Research

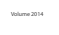

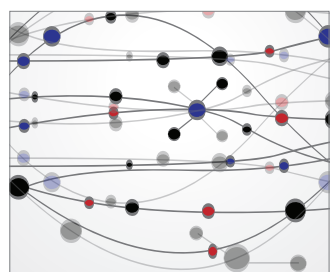

\section{The Scientific} World Journal
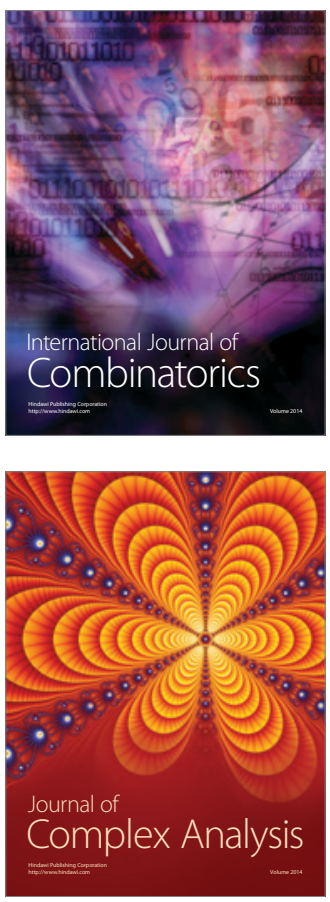

International Journal of

Mathematics and

Mathematical

Sciences
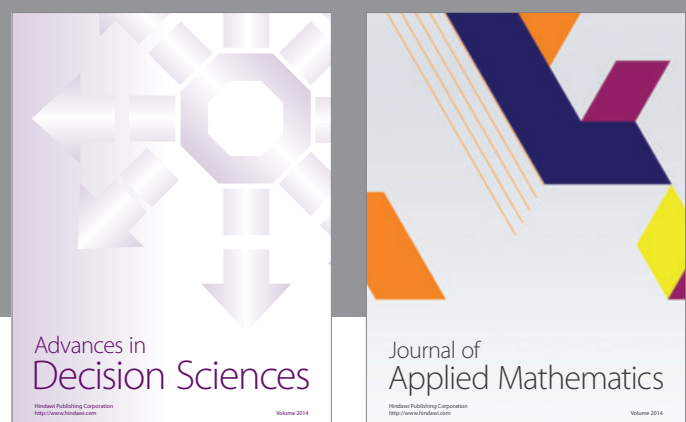

Journal of

Applied Mathematics
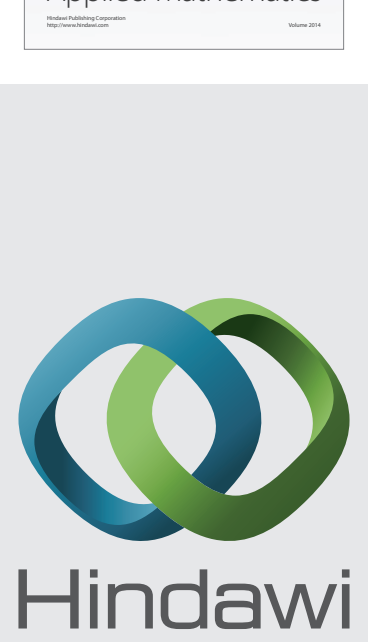

Submit your manuscripts at http://www.hindawi.com
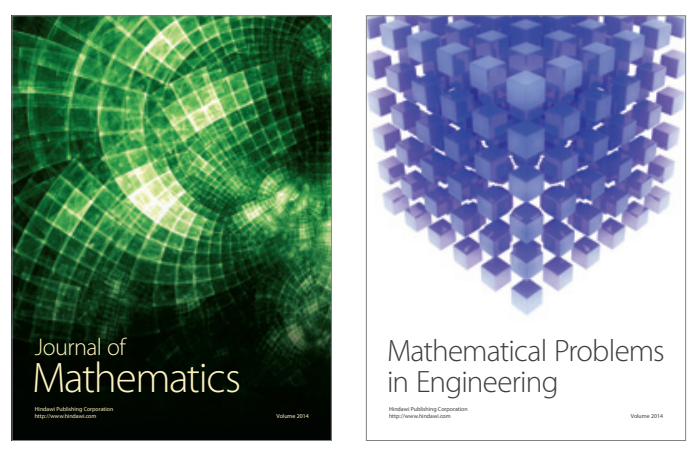

Mathematical Problems in Engineering
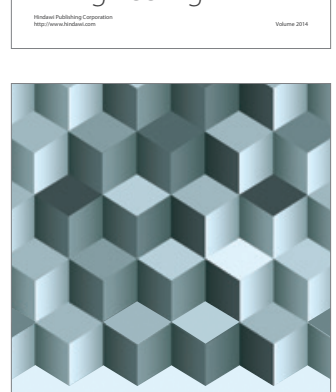

Journal of

Function Spaces
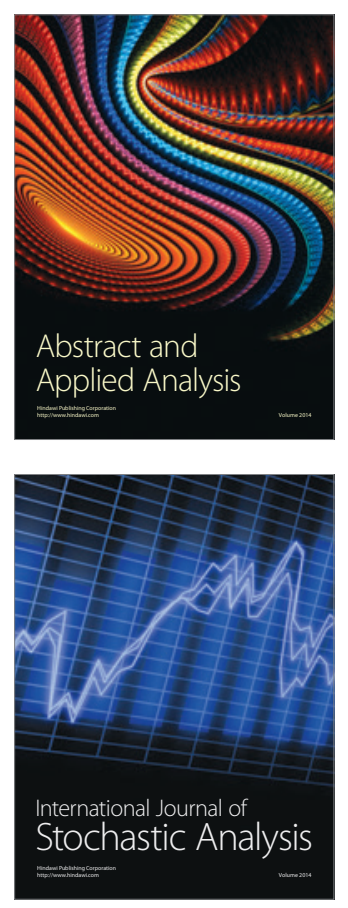

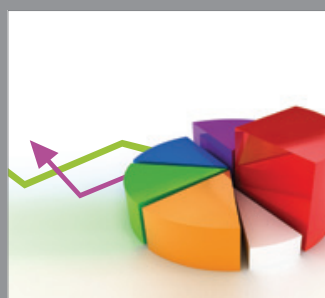

ournal of

Probability and Statistics

Promensencen
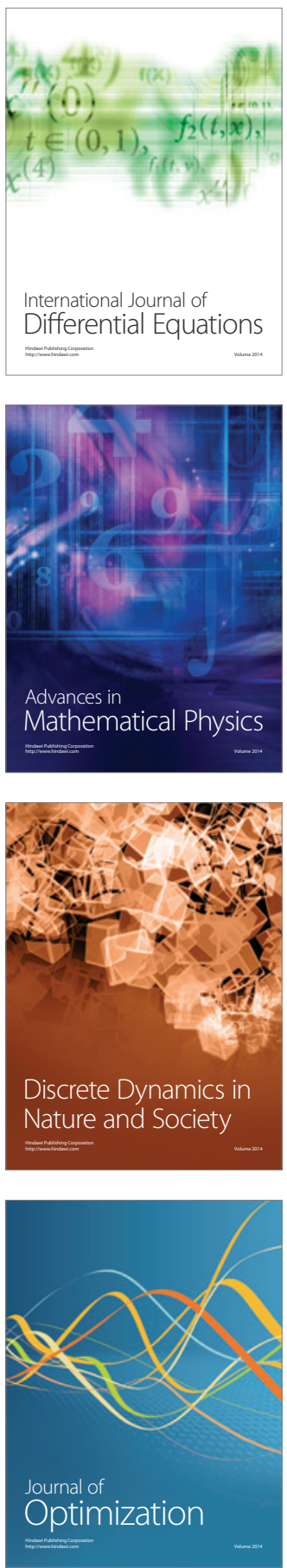